Article

\title{
GRK5 is an Essential Co-repressor for Cardiac Mineralocorticoid Receptor Antagonism induced by Finerenone but not Eplerenone
}

\author{
Victoria L. Desimine ${ }^{1,+}$, Jennifer Ghandour ${ }^{1}$, Natalie Cora ${ }^{1}$, Celina M. Pollard ${ }^{\text {, }}$, Rachel Valiente \\ 1, Krysten E. Ferraino ${ }^{1}$, Janelle Pereyra ${ }^{1}$, Daniela Pi Noa ${ }^{1}$, Yanelys Duarte 1, Yaimiry Martinez ${ }^{1}$, \\ Jennifer Maning ${ }^{1} \ddagger$, Barbara M. Parker ${ }^{1}$, Ava R. Brill ${ }^{1}$, Valentina Guidi ${ }^{2}$, Beatrix Aukszi ${ }^{2}$, and \\ Anastasios Lymperopoulos 1 ,* \\ 1 Laboratory for the Study of Neurohormonal Control of the Circulation, Department of Pharmaceutical \\ Sciences, College of Pharmacy, Nova Southeastern University, Fort Lauderdale, FL 33328, USA; \\ vd359@mynsu.nova.edu (V.L.D.); jg2901@mynsu.nova.edu (J.G.); nc1174@mynsu.nova.edu (N.C.); \\ cp1743@mynsu.nova.edu (C.M.P.); rv475@mynsu.nova.edu (R.V.); kf713@mynsu.nova.edu (K.E.F.); \\ jp2798@mynsu.nova.edu (J.P.); dp1726@mynsu.nova.edu (D.P.N.); yd208@mynsu.nova.edu (Y.D.); \\ ym359@mynsu.nova.edu (Y.M.); jm3706@mynsu.nova.edu (J.M.); barbaramparker@gmail.com (B.M.P.); \\ avabrill@gmail.com (A.R.B.) \\ 2 Department of Chemistry and Physics, Halmos College of Natural Sciences and Oceanography, Nova \\ Southeastern University, Fort Lauderdale, FL 33328, USA; vg505@mynsu.nova.edu (V.G.); ba285@nova.edu \\ (B.A.) \\ * Correspondence: al806@nova.edu; Tel.: +1-954-262-1338; Fax: +1-954-262-2278 \\ † Present address: James A. Haley Veterans' Hospital, Tampa, FL 33612, USA. \\ $\ddagger$ Present address: Jackson Memorial Hospital, Miami, FL 33136, USA.
}

\begin{abstract}
:
Background: In the heart, aldosterone (Aldo) binds the mineralocorticoid receptor (MR) to exert damaging, adverse remodeling-promoting effects. We recently showed that $G$ protein-coupled receptor (GPCR)-kinase (GRK)-5 blocks the cardiac MR by directly phosphorylating it, thereby repressing its transcriptional activity. MR antagonist (MRA) drugs block the cardiac MR reducing morbidity and mortality of advanced human heart failure. Non-steroidal MRAs, such as finerenone, may provide better cardio-protection against Aldo than classic, steroidal MRAs, like spironolactone and eplerenone. Herein, we sought to investigate potential differences between finerenone and eplerenone at engaging GRK5-dependent cardiac MR phosphorylation and subsequent blockade.
\end{abstract}

Methods: We used the cardiomyocyte cell line $\mathrm{H} 9 \mathrm{c} 2$ and neonatal rat ventricular myocytes (NRVMs).

Results: GRK5 phosphorylates the MR in H9c2 cardiomyocytes in response to finerenone but not to eplerenone. Unlike eplerenone, finerenone alone potently and efficiently suppresses cardiac MR transcriptional activity, thus displaying inverse agonism. GRK5 is necessary for finerenone's inverse agonism, since GRK5 genetic deletion renders finerenone incapable of blocking cardiac MR transcriptional activity. Eplerenone alone does not fully suppress cardiac MR basal activity regardless of GRK5 expression levels. Finally in NRVMs, GRK5 is necessary for the anti-apoptotic and anti-fibrotic effects of both finerenone and eplerenone against Aldo, as well as for the higher efficacy and potency of finerenone at blocking Aldo-induced apoptosis and fibrosis.

Conclusions: Finerenone, but not eplerenone, induces GRK5-dependent cardiac MR inhibition, which underlies, at least in part, its higher potency and efficacy, compared to eplerenone, as an MRA in the heart. GRK5 acts as a co-repressor of the cardiac MR and is essential for efficient MR antagonism in the myocardium. 
Keywords: aldosterone; apoptosis; cardiac myocyte; eplerenone; fibrosis; finerenone; G protein-coupled receptor kinase (GRK)-5; mineralocorticoid receptor; mineralocorticoid receptor antagonist (MRA); signal transduction

\section{Introduction}

Aldosterone (Aldo) is one of several cardio-toxic hormones, whose elevated circulating levels significantly confound and aggravate heart disease, including hypertension and chronic heart failure (CHF) [1-4]. The mineralocorticoid receptor (MR), a cytosolic transcription factor that, upon activation, translocates to the nucleus to activate gene transcription, is the main receptor mediating Aldo's adverse remodeling effects in the failing heart [1,5]. GRK2 and GRK5 are the most abundant cardiac G protein-coupled receptor (GPCR)-kinase (GRK) isoforms. Both phosphorylate GPCRs but also non-GPCR substrates [6-10]. We recently showed that GRK5 blocks the cardio-toxic MR-dependent effects of aldosterone in the heart by directly phosphorylating the cardiac MR and inhibiting its transcriptional activity [11].

MR antagonist (MRA) drugs are beneficial in human advanced CHF thanks to their blockade of the MR in various cardiovascular tissues, including in cardiomyocytes and cardiac fibroblasts [3,12]. Novel, non-steroidal MRAs, such as finerenone, may provide better cardio-protection against aldosterone's cardio-toxic actions than the classic steroidal MRAs, such as sprironolactone and eplerenone $[13,14]$. Indeed, finerenone was recently shown to be a more potent and efficacious inverse agonist at the MR, compared to eplerenone, in terms of cardiac fibrosis/adverse remodeling attenuation [15]. This prompted us to investigate the effects of these two MRAs on GRK5-dependent cardiac MR phosphorylation and subsequent suppression, in an effort to delineate potential molecular mechanisms underlying their differences in cardiac MR blocking efficacy. Indeed, we found that finerenone, but not eplerenone, promotes the inhibitory action of GRK5 on cardiac MR, which may underlie finerenone`s significantly greater efficacy/potency as an inverse agonist at this receptor. Moreover, GRK5 is necessary for both MRA drugs' cardioprotective actions against Aldo in cardiac myocytes.

\section{Materials and Methods}

All drugs/chemicals were from Sigma-Aldrich (St. Louis, MO, USA), except for finerenone (BAY94-8862) which was purchased from MedKoo Biosciences, Inc. (Cat. \#319698, Morrisville, NC, USA).

\subsection{Cell Culture, Viruses, and Transfections}

The H9c2 rat cardiomyoblast cell line was purchased from American Type Culture Collection (Manassas, VA, USA) and cultured as previously described [11,16,17]. Neonatal rat ventricular myocytes (NRVMs) were isolated and cultured, as previously described [18]. Recombinant lentiviruses encoding for wild-type full-length GRK5 or for empty vector (control) (OriGene Technologies, Rockville, MD, USA) were propagated and purified via $\mathrm{CsCl}$ density gradient ultracentrifugation, as described previously [11,19]. For CRISPR/Cas9-mediated GRK5 gene deletion, a gRNA sequence was custom-synthesized by Sigma-Aldrich (target ID: RN0000391809, target sequence: 5'-GTGGTTTGAATTTATGCGG-3') and incorporated into a lentiviral vector (Sigma-Aldrich). Along with negative control CRISPR lentiviral particles (CNCV, Cat \#CRISPR12V-1EA, Sigma-Aldrich), this lentivirus was also propagated and purified through cesium chloride density gradient ultracentrifugation.

\subsection{Immunoprecipitation (IP) and Western Blotting}

Cell extracts were prepared, as described previously [11,20], in a 20-mM Tris pH 7.4 buffer containing $137 \mathrm{mM} \mathrm{NaCl}, 1 \%$ Nonidet P-40, 20\% glycerol, $10 \mathrm{mM}$ phenylmethylsulfonylfluoride 
(PMSF), $1 \mathrm{mM} \mathrm{Na} \mathrm{VO}_{4}, 10 \mathrm{mM} \mathrm{NaF}, 2.5 \mu \mathrm{g} / \mathrm{mL}$ aprotinin, and $2.5 \mu \mathrm{g} / \mathrm{mL}$ leupeptin. Protein concentration was determined (Pierce BCA Protein Assay Kit, Thermo Scientific, Waltham, MA, USA), and equal amounts of protein per sample were used for IP or western blotting. MR was immunoprecipitated by overnight incubation of extracts with an anti-MR antibody (\#ab62532; Abcam, Cambridge, MA, USA), attached to Protein A/G-Sepharose beads (Sigma-Aldrich). The IPs were then subjected to immunoblotting for GRK5 (\#sc-565; Santa Cruz Biotechnology, Santa Cruz, CA, USA) or for phosphoserine (\#AB1603; Millipore-Sigma, Burlington, MA, USA) to measure the pSer content of the immunoprecipitated MR. Finally, an anti-glyceraldehyde 3-phosphate dehydrogenase (GAPDH) antibody (\#sc-25778; Santa Cruz Biotechnology) was used to control for protein loading. All immunoblots were revealed by enhanced chemiluminescence (ECL, Life Technologies, Grand Island, NY, USA) and visualized in the FluorChem E Digital Darkroom (Protein Simple, San Jose, CA, USA), as described previously [21].

\subsection{Luciferase Reporter Activity Assay}

Luciferase reporter activity assay was performed, as described previously, by transfecting the cells with the LightSwitch ${ }^{\mathrm{TM}}$ luciferase reporter gene vector under the influence of the MR promoter (Active Motif, Inc., Carlsbad, CA, USA) [11]. The measurements were done the next day with the manufacturer's LightSwitch ${ }^{\mathrm{TM}}$ assay kit and according to the manufacturer`s instructions.

\subsection{TUNEL}

Terminal deoxynucleotidyl transferase-mediated dUTP nick-end labeling (TUNEL) assay to measure apoptotic cell death was done as described [22]. Briefly, cells were fixed with $10 \%$ neutral buffered formalin, embedded in paraffin, and sectioned at 5- $\mu$ m thickness. DNA fragmentation was detected in situ in deparaffinized sections using the ApopTag peroxidase in situ apoptosis detection Kit (Millipore-Sigma) and according to the manufacturer's instructions. The total number of nuclei was determined by manual counting of $4^{\prime}, 6^{\prime}$-diamidino-2-phenylindole (DAPI)-stained nuclei in six random fields per section. All terminal deoxynucleotidyl transferase-mediated dUTP nick end-labeling (TUNEL)-positive nuclei were counted in each section.

\subsection{Real-Time PCR}

Real-time PCR for rat plasminogen activator inhibitor (PAI)-1 and rat fibronectin mRNA levels in total RNA isolated from NRVMs was done as described previously [16]. Briefly, quantitative real-time PCR was performed using a MyIQ Single-Color Real-Time PCR detection system (Bio-Rad Laboratories, Hercules, CA, USA) using SYBR Green Supermix (Bio-Rad) and $100 \mathrm{nM}$ of gene-specific oligonucleotides. Quantification of mRNA included normalization to 18s rRNA levels. No bands were seen in control reactions in the absence of reverse transcriptase. Primer pairs used were: 5'-TTCCTCCACAGCCATTCTAGTCT-3' and 5'-GAAAGGATCGGTCTAAAACCATCTC-3' for PAI-1; 5'-CGAGGTGACAGAGACCACAA-3' and 5'-CTGGAGTCAAGCCAGACACA-3` for fibronectin; and 5'-TCGATGCTCTTAGCTGAGTG-3` and 5'-TGATCGTCTTCGAACCTCC-3` for $18 \mathrm{~S}$ rRNA.

\subsection{Statistical Analysis}

Student's $t$ test and one- or two-way ANOVA with Bonferroni test were used for statistical comparisons, unless otherwise indicated. For multiple group analyses, Dunnett's test with SAS version 9 software (Cary, NC, USA) was also used. A $p$ value of $<0.05$ indicated statistical significance.

\section{Results}

3.1. Finerenone, but not eplerenone, induces GRK5-dependent cardiac MR phosphorylation.

We recently reported that GRK5 selectively phosphorylates and inhibits the cardiac MR [11]. Based also on recent evidence suggesting greater potency for finerenone, compared to eplerenone, at inhibiting the cardiac MR and its downstream fibrosis [15], we hypothesized, in the present study, that the higher efficacy/potency of finerenone over eplerenone might be due (at least in part) to 
differences in modulation of the GRK5 inhibitory action on the cardiac MR. Thus, in a first series of experiments, we overexpressed or knocked out (via CRISPR) GRK5 in H9c2 cardiac myocytes (Figure 1A), which endogenously express both GRK5 and MR [11,23], and checked for the effects of the two MRA drugs on MR serine phosphorylation. GRK5, being a Ser/Thr kinase, likely phosphorylates multiple Ser and Thr residues of the MR protein, with phosphorylations of Ser601 and Ser843 (in the human orthologue sequence), in particular, resulting in significant functional inhibition of the MR, courtesy of cytosolic retention and transcriptional activity suppression, respectively [24,25]. After preliminary concentration-response experiments (not shown), and based on the associated literature, we chose a $10 \mu \mathrm{M}$ concentration for both drugs throughout the experiments of our study, as this concentration $(10 \mu \mathrm{M})$ is quite close to both drugs 'effective IC 50 values $[12,15]$.

A

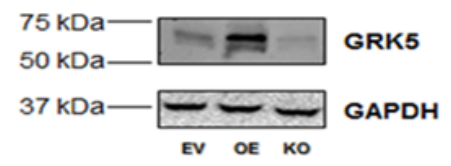

B

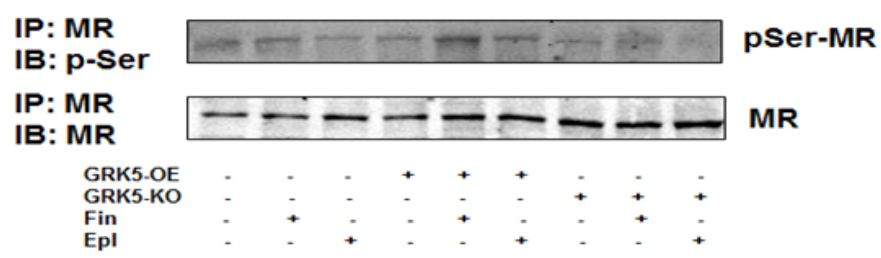

160
C

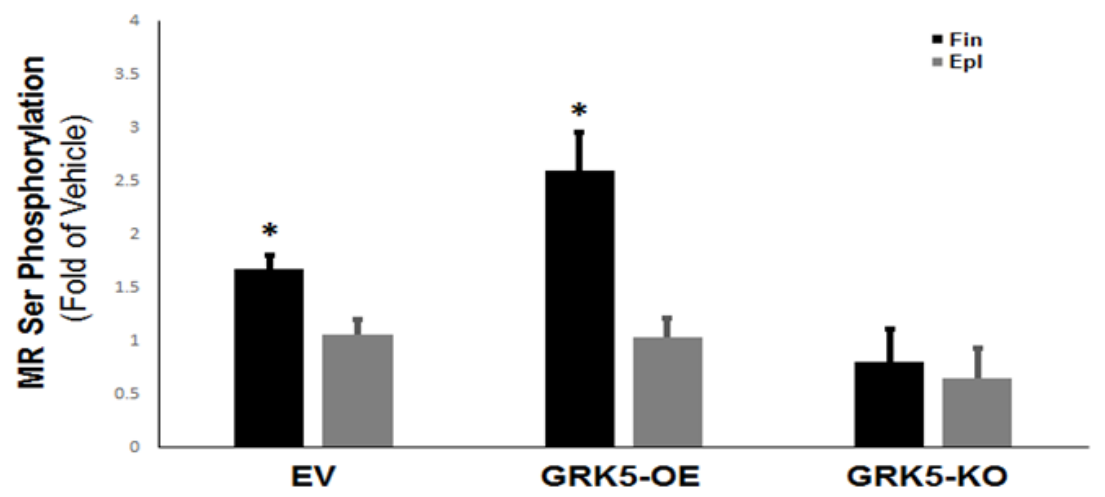

Figure 1. GRK5 phosphorylates the cardiac MR in response to finerenone but not to eplerenone. (A) Western blotting to confirm GRK5 overexpression (OE) with a wild type GRK5-encoding lentivirus or deletion (KO) via a GRK5-targeting CRISPR lentivirus in H9c2 cardiomyocytes. GAPDH blotting is also shown as loading control. EV: empty vector mock virus-transfected (control) cells. (B, C) Western blotting for the phosphoserine content of the $\mathrm{MR}$ in response to $10 \mu \mathrm{M}$ finerenone (Fin) or $10 \mu \mathrm{M}$ eplerenone (Epl) in GRK5-overexpressing (GRK5-OE) or in GRK5-KO or in control, empty virus (EV)-infected H9c2 cells. IP: Immunoprecipitation; IB: Immunoblotting. Representative blots are shown in (B) and the densitometric quantitation of three independent experiments in $(\mathbf{C}) .{ }^{*}, \mathrm{p}<0.05$, vs. Epl; $\mathrm{n}=3$ independent experiments performed in duplicate per cell clone/treatment.

As shown in Figures 1B \& 1C, finerenone led to much higher phosphorylation (pSer content) of the MR than eplerenone did in control H9c2 cardiomyocytes (mock virus-EV lanes). This finerenone-induced MR phosphorylation was significantly enhanced upon GRK5 overexpression but essentially abrogated in GRK5-depleted H9c2 cardiomyocytes (Figures 1B \& 1C). Notably, eplerenone essentially failed to elicit any appreciable MR Ser phosphorylation in H9c2 cardiomyocytes (Figures 1B \& 1C), irrespective of GRK5 expression levels [eplerenone-induced phosphorylation: $1.2 \pm 0.25$-fold of vehicle in EV cells; $1.23 \pm 0.27$-fold of vehicle in GRK5-OE cells; 
$0.6 \pm 0.55$-fold of vehicle in GRK5-KO cells; i.e. non-significant vs. vehicle, in all three clones at $\mathrm{p}=0.05$ $(\mathrm{n}=3)$; Figure 1C]. Although we cannot account for the potential of some extent of $\mathrm{Thr}$ phosphorylation of the MR induced by the two drugs, these results strongly suggest that only finerenone (not eplerenone) induces GRK5-mediated phosphorylation of the MR in H9c2 cardiac myocytes.

\subsection{GRK5 is essential for finerenone's inverse agonism at the cardiac MR.}

Since GRK5-induced phosphorylation translates into transcriptional repression of the cardiac MR [11], we next examined the impact of the finerenone-induced, GRK5-mediated MR phosphorylation on the transcriptional activity of the receptor.

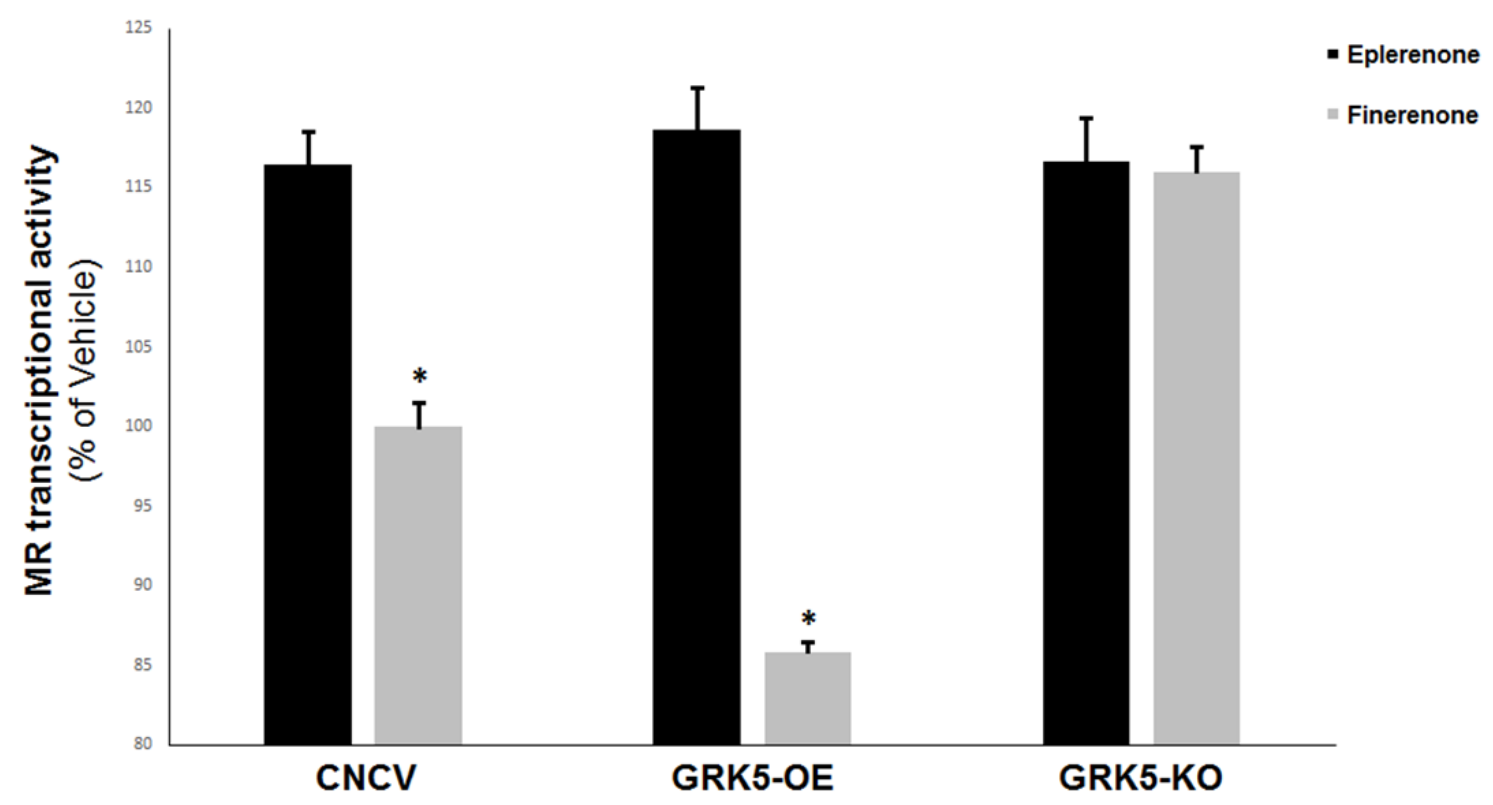

Figure 2. GRK5 inhibits the cardiac MR in response to finerenone but not to eplerenone. Transcriptional activity of the MR in response to either $10 \mu \mathrm{M}$ eplerenone or $10 \mu \mathrm{M}$ finerenone in H9c2 cardiomyocytes overexpressing GRK5 (GRK5-OE) or having GRK5 genetically deleted via CRISPR (GRK5-KO). Neither agent was able to suppress MR basal transcriptional activity in GRK5-KO cells. CNCV: CRISPR negative control virus-infected control cells. ${ }^{*}, \mathrm{p}<0.05$, vs. eplerenone; $\mathrm{n}=5$ independent measurements per cell clone/treatment performed in triplicate.

In contrast with eplerenone, finerenone lacks agonist activity at the MR in control (CNCV) H9c2 cardiomyocytes, i.e. no increase in MR basal transcriptional activity (in the absence of Aldo) is observed with finerenone (Figure 2). In the absence of GRK5 however, finerenone loses the ability to keep the MR transcriptionally inactive, i.e. the MR displays significant basal activity in GRK5-KO H9c2 cardiomyocytes (Figure 2). Upon GRK5 overexpression, this picture is reversed, i.e. finerenone acts as potent inverse agonist at the MR, markedly suppressing MR basal transcriptional activity in GRK5-overexpressing (GRK5-OE) cardiomyocytes (Figure 2). In contrast, eplerenone allows for substantial MR basal transcriptional activity, regardless of GRK5 expression levels (Figure 2). Taken together, these results indicate that GRK5 is essential for finerenone`s inverse agonism at the cardiac MR, while eplerenone is essentially a partial agonist (mixed agonist/antagonist) at this receptor in the heart, a finding consistent with the literature [12,15]. GRK5 is unable to affect eplerenone's 
208

actions on the cardiac MR, probably because this MRA agent cannot induce the inhibitory phosphorylation of this receptor by GRK5 in cardiac myocytes (see above, Figure 1).

3.3. GRK5 is essential for MRA-dependent anti-apoptosis in the heart and for finerenone's advantage over eplerenone towards this effect.

Since H9c2 cells are not an entirely physiologically relevant cardiomyocyte cell line (for instance, they are incapable of contraction) and their endogenous MR expression levels might reportedly be low [15], we switced to the much more physiologically relevant NRVMs, a bona fide cardiac myocyte cell model, for the rest of our experiments, in which we compared the cardio-protective efficacies of the two MRA drugs against the deleterious actions of Aldo.

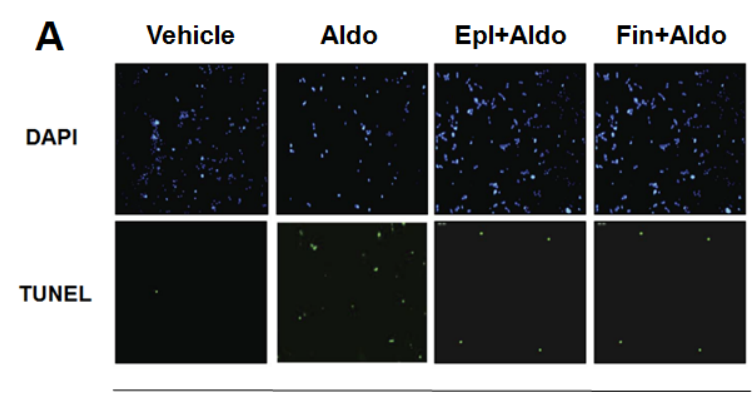

EV

B

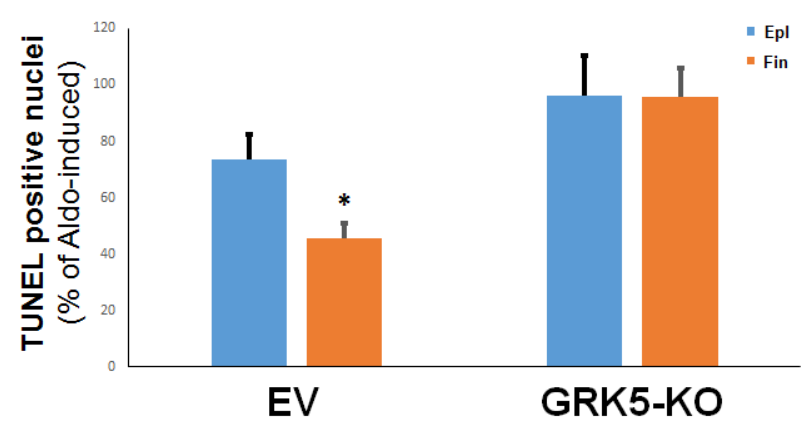

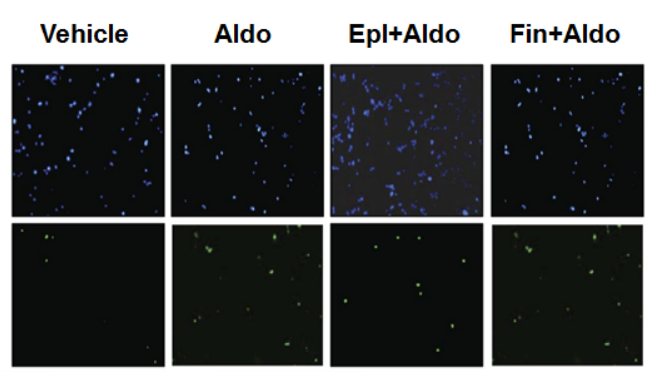

GRK5-KO

Figure 3. Comparison of the anti-apoptotic efficacies of finerenone vs. eplerenone in aldosterone-treated cardiomyocytes in the presence or absence of GRK5. (A, B) Apoptotic cell death in control, empty vector (mock) virus-infected (EV) NRVMs or in NRVMs having GRK5 genetically (CRISPR lentivirus-mediated) deleted (GRK5-KO) and treated with $100 \mathrm{nM}$ aldosterone (Aldo) alone or in the presence of $10 \mu \mathrm{M}$ eplerenone (Epl+Aldo) or $10 \mu \mathrm{M}$ finerenone (Fin+Aldo) for $24 \mathrm{hrs.} \mathrm{*}$, $\mathrm{p}<0.05$, vs. Epl; $\mathrm{n}=4$ independent experiments per transfection/treatment. No inhibition of Aldo-induced apoptosis could be detected with either drug in GRK5-KO cells. (C) Western blotting in NRVM total protein extracts to confirm the CRISPR-mediated genetic deletion of GRK5 in the GRK5-KO (KO) NRVMs. GAPDH is also shown as loading control.

As shown in Figures 3A \& 3B, finerenone was much more effective than eplerenone at suppressing Aldo-induced apoptosis in control (EV) NRVMs. However, upon GRK5 genetic deletion from NRVMs (Figure 3C), the two drugs similarly failed to block Aldo-induced apoptosis (Figures 3A \& 3B). This strongly suggests that GRK5 is essential for the anti-apoptotic effects of MRAs against Aldo in the heart, as well as for the better antagonistic efficacy of finerenone vs. eplerenone against

234 Aldo-induced cardiac apoptosis. 
235

236

237

238

239

240

241

242

243

244

245

246

3.4. GRK5 is essential for MRA-dependent anti-fibrotic effects in the heart and for finerenone's advantage over eplerenone towards this effect.

In addition to Aldo-induced apoptosis, we also compared the two MRAs in terms of Aldo-induced fibrosis inhibition in isolated and cultured NRVMs. Assessment of Aldo-dependent mRNA induction of two major pro-fibrotic stimuli, PAI-1 and fibronectin, both of which are immediate/early MR-responsive genes [1,3,16], revealed that finerenone was more effective than eplerenone at suppressing both PAI-1 (Figure 4A) and fibronectin (Figure 4B) mRNA inductions by Aldo in control NRVMs. Again however, neither drug was effective at all when GRK5 was absent (Figures 4A \& 4B, compare with GRK5-KO bars).
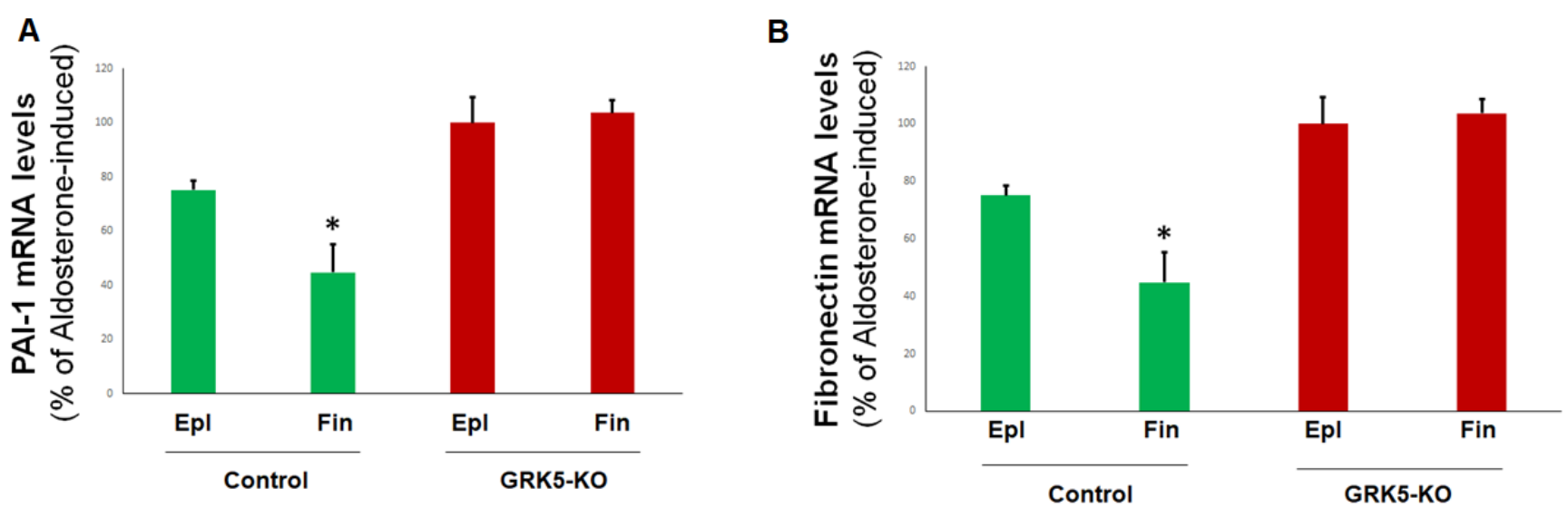

Figure 4. Comparison of the anti-fibrotic efficacies of finerenone vs. eplerenone in aldosterone-treated cardiomyocytes in the presence or absence of GRK5. mRNA levels of PAI-1 (A) and fibronectin (B), in response to a 2-hr-long treatment of $100 \mathrm{nM}$ aldosterone in the presence of either $10 \mu \mathrm{M}$ eplerenone (Epl) or $10 \mu \mathrm{M}$ finerenone (Fin) in control (mock, empty vector CRISPR lentivirus-infected) or in GRK5-KO (rat GRK5-targeting CRISPR lentivirus-infected) NRVMs. 18S rRNA levels were used for normalization of the results. ${ }^{*}, \mathrm{p}<0.05$, vs. Epl; $\mathrm{n}=3$ independent measurements per cell clone/treatment performed in triplicate.

Thus, GRK5 is essential not only for the anti-apoptotic, but also for the anti-fibrotic effects of MRAs in cardiac myocytes. In addition, finerenone displays superior cardio-protection (anti-apoptosis, anti-fibrosis) against Aldo versus eplerenone, thanks to its promotion of cardiac GRK5-dependent MR inhibition, which eplerenone is incapable of eliciting (see above, Figures 1 \& 2).

\section{Discussion}

In the present study, we report that finerenone is a more potent and efficacious cardiac MR blocker than eplerenone, thanks, at least in part, to stimulation of GRK5-dependent cardiac MR phosphorylation, which eplerenone is incapable of inducing (Figure 5). This non-canonical effect of GRK5 on the cardiac MR is essential for efficient blockade of Aldo's deleterious actions in the heart, such as apoptosis, fibrosis, and probably other adverse remodeling-associated effects (Figure 5). Therefore, GRK5-dependent inhibitory phosphorylation is a key molecular mechanism for cardiac MR inverse agonism and needs to be considered in the design \& development of novel, more effective MRA drugs for heart disease (e.g. CHF, hypertension, renal insufficiency, etc.) treatment. The MR has long been established as an important molecular culprit in heart disease progression [1-5], including a recent study in transgenic mice showing that, unlike its closely related glucocorticoid receptor, the MR promotes cardiac dysfunction even in the absence of a cardiac insult or injury [26]. Indeed, the well-documented deleterious effects of the cardiac MR have provided the 
272 pharmacological basis for the use of MRA drugs in advanced stage human CHF and other heart 273 diseases [1-5,27,28]. The MRA drug class, which began with the approval and marketing of 274 spironolactone more than 60 years ago, now encompasses several agents, with some already in 275 clinical use and some in clinical trials. The MRAs are broadly divided to traditional, steroidal 276 MRAs, like spironolactone and eplerenone currently in clinical use, and later generation, 277 non-steroidal agents. Among the latter is finerenone (formerly BAY 94-8862), a third generation, non-steroidal, dihydropyridine-derived MRA currently in phase III clinical trials [3,12].

Despite being very potent and effective aldosterone antagonists with salutary effects in the heart and kidneys, the currently available steroidal MRAs are hampered by several limiting side effects, most prominent of which are hyperkalemia, renal function deterioration, and gynecomastia. These are generally thought to be due to their binding to other types of steroid receptors (e.g. estrogen receptor, glucocorticoid receptor, etc.) exactly because of their steroidal structure $[3,12,29]$. Thus, non-steroidal MRAs have been developed, currently headlined by finerenone. Finerenone has shown advantageous pharmacological and therapeutic profiles, compared to the steroidal MRAs. It has demonstrated improved therapeutic properties in heart failure animal models in head-to-head comparisons with eplerenone $[12,15]$ and leads to bigger improvements in HFrEF (heart failure with reduced ejection fraction) confounded by diabetes or chronic kidney disease [12,14]. In addition to its much higher selectivity for the MR over other steroid receptors, finerenone is also at least one log scale more potent at MR antagonism than eplerenone and spironolactone, both of which are competitive MR antagonists [3]. Furthermore, finerenone displays inverse agonist activity at the MR, whereas the steroidal MRAs are only partial MR antagonists [3,12]. This means that, depending on the activity status of the MR, spironolactone and eplerenone may actually promote the activity of the MR rather than inhibiting it $[12,14,15]$. In other words, eplerenone inhibits the MR when the receptor is activated by Aldo but it may actually promote the activity of the MR when bound alone to the receptor (in the absence of Aldo). Finerenone, thanks to the non-steroidal nature of its structure, appears to be devoid of any agonist activity at the MR and thus, has strong potential to provide better cardiovascular and renal outcomes, especially in diseases severely affected by hyperaldosteronism.

One of the most important parameters affecting the selectivity of a particular MRA for the MR versus other steroid receptors, as well as tissue specificity for MR antagonism (inhibition of the cardiac MR versus inhibition of the MR in other tissues), is the identity/identities of the receptor's co-factors activated or repressed by the MRA agent, which ultimately affects the MRA drug's potency \& efficacy $[1,3,15,25]$. In other words, how good a particular MRA is at blocking the cardiac MR depends strongly on which co-activators of the MR the drug inhibits and/or which co-repressors of the MR it activates inside the cardiac myocyte [3]. Indeed, a recent study in mice reported much higher potency and inverse agonism of finerenone, relative to eplerenone, in terms of cardiac fibrosis suppression and suggested that the pharmacological difference between these two MRAs was probably due to differential cardiac MR co-factor regulation/engagement [15]. We recently uncovered that GRK5 is an important co-repressor of the cardiac MR, via its direct binding to, and phosphorylation of the MR that results in cytosolic retention of the phosphorylated receptor and thus, MR transcriptional repression [11]. Our present data strongly suggest that finerenone selectively activates this kinase in cardiac myocytes to potently inhibit/repress the cardiac MR. In contrast, eplerenone is incapable of this action (GRK5 activation) and thus, is a much weaker MR antagonist in the myocardium.

There are a few very important questions emanating from our present work that await delineation in future studies. First, does finerenone activate GRK5 to suppress MR activity only in the heart or in other tissues, as well (e.g. kidneys)? Another critical question is whether this property is shared by other non-steroidal MRAs or it is specific to finerenone. Finally, there is also the obvious mechanistic question of how exactly finerenone, not known to be a GPCR agonist, induces GRK5, normally activated by a GPCR, such as the $\beta_{2}$-adrenergic receptor (Figure 5) [8,11], to phosphorylate and inhibit the MR in the cytosol of a cardiac myocyte. Nevertheless, these salient questions will be the focus of our future investigations, along with our already ongoing efforts to map the specific 
phosphorylation sites of GRK5 on the human MR protein and to characterize the functional impact for the receptor of each one of them.

\section{Cardiac myocyte}

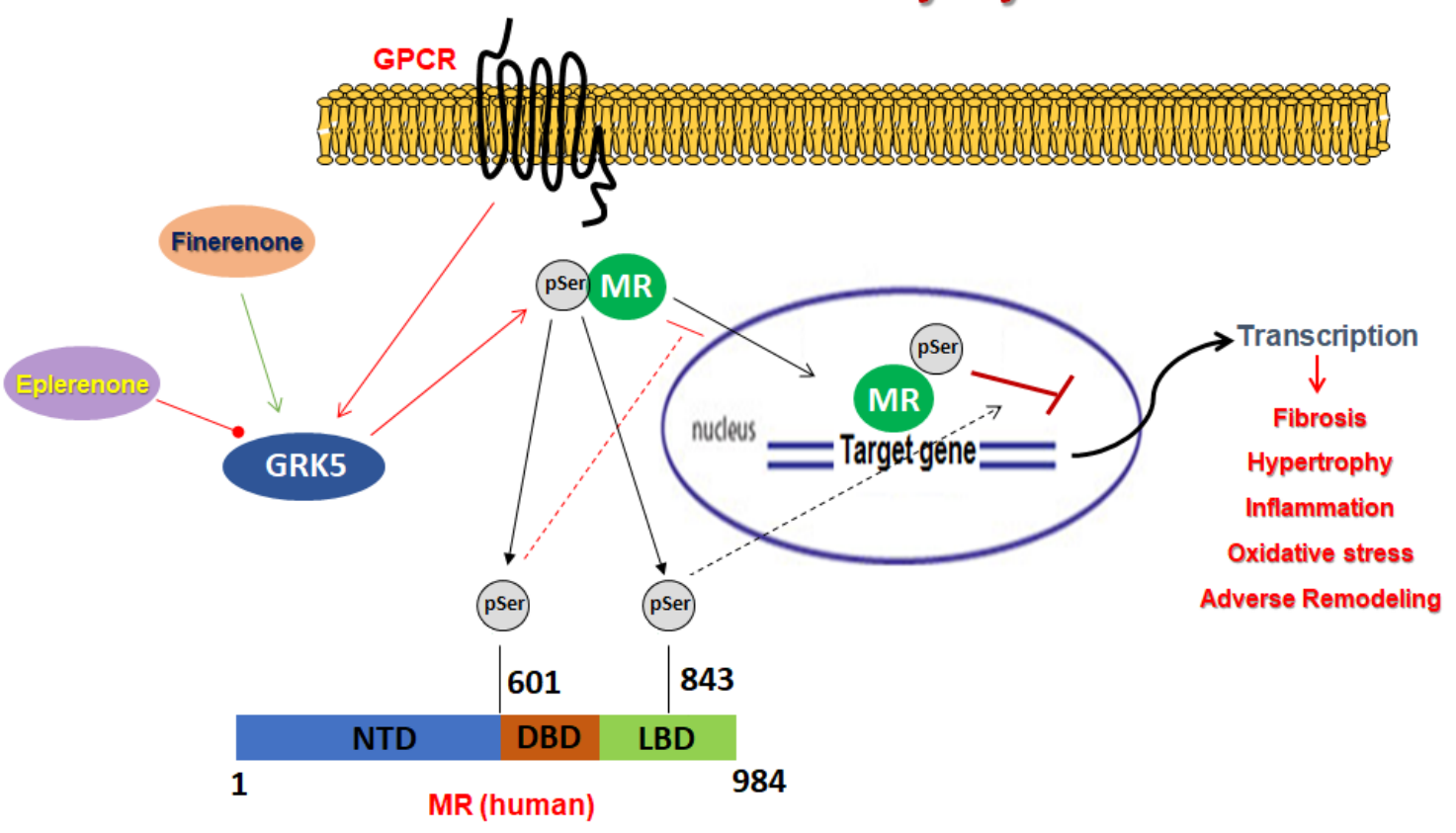

Figure 5. Schematic illustration of the differential effects of finerenone vs. eplerenone on GRK5-dependent repression of the cardiac MR. Finerenone, unlike eplerenone, stimulates GRK5 to phosphorylate the MR. The two main (putative) GRK5 phosphorylation sites on the human MR protein, Ser601 \& Ser843, are highlighted, along with their functional impacts for the MR (pSer601 blocks nuclear translocation; pSer843 suppresses Aldo-induced transcriptional activity) [24,30]. GPCR: G protein-coupled receptor; NTD: N-terminal domain; DBD: DNA-binding domain; LBD: Ligand-binding domain; pSer: Phosphoserine. See text for more details and for all other molecular acronyms`descriptions.

In summary, our present study reinforces the emerging and therapeutically very intriguing notion that GRK5, acting as a cardiac MR co-repressor in this instance, may actually be beneficial in the myocardium [11,31-33], contrary to its counterpart GRK2 that is generally considered deleterious in the heart $[7,10]$. Importantly, we have identified GRK5 as a potential co-factor of the cardiac MR that is differentially regulated by finerenone and eplerenone, which may underlie the higher potency/efficacy (and inverse agonism) of finerenone at the MR. To our knowledge, cardiac GRK5 is the first such MR co-factor to be shown as differentially modulated/stimulated among different individual MRA drugs. Finally, from the therapeutic standpoint, we provide evidence that GRK5 is indispensable for MRAs' cardioprotective actions against Aldo (e.g. anti-apoptosis, anti-fibrosis) and, importantly, this applies to both steroidal (eplerenone) and non-steroidal (finerenone) MRA agents alike.

\section{Conclusions}

Cardiac GRK5 is an essential mediator of the general cardio-protection afforded by MRA drugs against the cardio-toxic effects of excess Aldo, e.g. during CHF and other chronic cardiac diseases. This is due to the inhibitory phosphorylation GRK5 performs on the cardiac MR. This non-canonical (given the substrate is not a GPCR), co-repressor effect of GRK5 on cardiac MR is also (at least partly) 
352 responsible for the inverse agonism properties of finerenone at this receptor that bestow this non-steroidal MRA with superior potency and efficacy, compared to eplerenone, at protecting the heart against the damaging effects of Aldo. Finally, since GRK5 is a co-repressor of the MR, at least in the myocardium, its stimulation (or potentiation) should be a desired property of every novel MRA drug designed and developed for improved cardiovascular pharmacotherapy.

Author Contributions: V.L.D., J.G., N.C., C.M.P., R.V., K.E.F., J.P., D.P.N., Y.D., Y.M., J.M., B.M.P., A.R.B., and V.G. performed all experiments and assisted with data analysis. B.A. contributed to the writing of the manuscript. A.L. supervised the project, performed data analysis, provided funding for the study, and wrote the manuscript. All authors have read and approved the manuscript.

Funding: This study was supported in part by a Gateway to Research scholarship from the American Foundation for Pharmaceutical Education (AFPE) (to A.L.) and a Nova Southeastern University's President's Faculty Research \& Development Grant (to A.L. \& B.A.).

Acknowledgments: We thank Dr. Lina A. Shehadeh and members of her laboratory (University of Miami Miller School of Medicine, Miami, FL) for excellent technical assistance.

Conflicts of Interest: The authors declare no conflict of interest. The funders had no role in the design of the study; in the collection, analyses, or interpretation of data; in the writing of the manuscript, or in the decision to 


\section{References}

372

1. Parker, B.M.; Wertz, S.L.; Pollard, C.M.; Desimine, V.L.; Maning, J.; McCrink, K.A.; Lymperopoulos, A. Novel insights into the crosstalk between mineralocorticoid receptor and G protein-coupled receptors in Heart Adverse Remodeling and Disease. Int J Mol Sci 2018, 19, 3764. doi:10.3390/ijms19123764

2. Luther, J.M. Is there a new dawn for selective mineralocorticoid receptor antagonism? Curr Opin Nephrol Hypertens 2014, 23, 456-461. doi: 10.1097/MNH.0000000000000051

3. Lother, A.; Moser, M.; Bode, C.; Feldman, R.D.; Hein, L. Mineralocorticoids in the heart and vasculature: new insights for old hormones. Annu Rev Pharmacol Toxicol 2015, 55, $289-312$. doi:10.1146/annurev-pharmtox-010814-124302

4. Weber, K.T. Aldosterone in congestive heart failure. $N$ Engl J Med 2001, 345, 1689-1697. doi:10.1056/NEJMra000050

5. Mihailidou, A.S.; Funder, J.W. Nongenomic effects of mineralocorticoid receptor activation in the cardiovascular system. Steroids 2005, 70, 347-351. doi:10.1016/j.steroids.2005.02.004

6. Lymperopoulos, A.; Bathgate, A. Pharmacogenomics of the heptahelical receptor regulators G-protein-coupled receptor kinases and arrestins: the known and the unknown. Pharmacogenomics 2012, 13, 323-341. doi: 10.2217/pgs.11.178

7. Sato, P.Y.; Chuprun, J.K.; Schwartz, M.; Koch, W.J. The evolving impact of g protein-coupled receptor kinases in cardiac health and disease. Physiol Rev 2015, 95, 377-404. doi:10.1152/physrev.00015.2014

8. Komolov, K.E.; Benovic, J.L. G protein-coupled receptor kinases: Past, present and future. Cell Signal 2018, 41, 17-24. doi:10.1016/j.cellsig.2017.07.004

9. McCrink, K.A.; Brill, A.; Lymperopoulos, A. Adrenal G protein-coupled receptor kinase-2 in regulation of sympathetic nervous system activity in heart failure. World J Cardiol 2015, 7, 539-543. doi: 10.4330/wjc.v7.i9.539

10. Siryk-Bathgate, A.; Dabul, S.; Lymperopoulos, A. Current and future G protein-coupled receptor signaling targets for heart failure therapy. Drug Des Devel Ther 2013, 7, 1209-1222. doi:10.2147/DDDT.S35905

11. Maning, J.; McCrink, K.A.; Pollard, C.M.; Desimine, V.L.; Ghandour, J.; Perez, A.; Cora, N.; Ferraino, K.E.; Parker, B.M.; Brill, A.R.; Aukszi, B.; Lymperopoulos, A. Antagonistic Roles of GRK2 and GRK5 in Cardiac Aldosterone Signaling Reveal GRK5-Mediated Cardioprotection via Mineralocorticoid Receptor Inhibition. Int J Mol Sci 2020, 21, 2868. doi: 10.3390/ijms21082868

12. Kolkhof, P.; Jaisser, F.; Kim, S.Y.; Filippatos, G.; Nowack, C.; Pitt, B. Steroidal and Novel Non-steroidal Mineralocorticoid Receptor Antagonists in Heart Failure and Cardiorenal Diseases: Comparison at Bench and Bedside Handb Exp Pharmacol 2017, 243, 271-305. doi: 10.1007/164_2016_76

13. Sueta, D.; Yamamoto, E.; Tsujita, K. Mineralocorticoid Receptor Blockers: Novel Selective Nonsteroidal Mineralocorticoid Receptor Antagonists. Curr Hypertens Rep 2020, 22, 21. doi: 10.1007/s11906-020-1023-y

14. Rico-Mesa, J.S.; White, A.; Ahmadian-Tehrani, A.; Anderson, A.S. Mineralocorticoid Receptor Antagonists: a Comprehensive Review of Finerenone. Curr Cardiol Rep 2020, 22, 140 . doi: 10.1007/s11886-020-01399-7

15. Grune, J.; Beyhoff, N.; Smeir, E.; Chudek, R.; Blumrich, A.; Ban, Z.; Brix, S.; Betz, I.R.; Schupp, M.; Foryst-Ludwig, A.; Klopfleisch, R.; Stawowy, P.; Houtman, R.; Kolkhof, P.; Kintscher, U. Selective Mineralocorticoid Receptor Cofactor Modulation as Molecular Basis for Finerenone's Antifibrotic Activity. Hypertension 2018, 71, 599-608. doi: 10.1161/HYPERTENSIONAHA.117.10360

16. Pollard, C.M.; Desimine, V.L.; Wertz, S.L.; Perez, A.; Parker, B.M.; Maning, J.; McCrink, K.A.; Shehadeh, L.A.; Lymperopoulos, A. Deletion of Osteopontin Enhances $\beta_{2}$-Adrenergic Receptor-Dependent Anti-Fibrotic Signaling in Cardiomyocytes. Int J Mol Sci 2019, 20, 1396. doi: 10.3390/ijms20061396

17. McCrink, K.A.; Maning, J.; Vu, A.; Jafferjee, M.; Marrero, C.; Brill, Bathgate-Siryk, A.; Dabul, S.; Koch, W.J.; Lymperopoulos, A. $\beta$-Arrestin2 Improves Post-Myocardial Infarction Heart Failure via Sarco(endo)plasmic Reticulum $\mathrm{Ca}^{2+}$-ATPase-Dependent Positive Inotropy in Cardiomyocytes. Hypertension 2017, 70, 972-981. doi:10.1161/HYPERTENSIONAHA.117.09817

18. McCrink, K.A.; Brill, A.; Jafferjee, M.; Valero, T.R.; Marrero, C.; Rodriguez, M.M.; Hale, G.M.; Lymperopoulos, A. $\beta$ 1-adrenoceptor Arg389Gly polymorphism confers differential $\beta$-arrestin-binding tropism in cardiac myocytes. Pharmacogenomics 2016, 17, 1611-1620. doi: 10.2217/pgs-2016-0094

19. Pollard, C.M.; Ghandour, J.; Cora, N.; Perez, A.; Parker, B.M.; Desimine, V.L.; Wertz, S.L.; Pereyra, J.M.; Ferraino, K.E.; Patel, J.J.; Lymperopoulos, A. GRK2-Mediated Crosstalk Between $\beta$-Adrenergic and 
Angiotensin II Receptors Enhances Adrenocortical Aldosterone Production In Vitro and In Vivo. Int J Mol Sci 2020, 21, 574. doi: 10.3390/ijms21020574

20. Nguyen, K.; Kassimatis, T.; Lymperopoulos, A. Impaired desensitization of a human polymorphic $\alpha$ B-adrenergic receptor variant enhances its sympatho-inhibitory activity in chromaffin cells. Cell Commun Signal 2011, 9, 5. doi:10.1186/1478-811X-9-5

21. Salazar, N.C.; Vallejos, X.; Siryk, A.; Rengo, G.; Cannavo, A.; Liccardo, D.; De Lucia, C.; Gao, E.; Leosco, D.; Koch, W.J.; Lymperopoulos, A. GRK2 blockade with $\beta$ ARKct is essential for cardiac $\beta 2$-adrenergic receptor signaling towards increased contractility. Cell Commun Signal 2013, 11, 64. doi:10.1186/1478-811X-11-64

22. Lymperopoulos, A.; Rengo, G.; Zincarelli, C.; Kim, J.; Koch, W.J. Adrenal beta-arrestin 1 inhibition in vivo attenuates post-myocardial infarction progression to heart failure and adverse remodeling via reduction of circulating aldosterone levels. J Am Coll Cardiol 2011, 57, 356-365. doi:10.1016/j.jacc.2010.08.635

23. Ashton, A.W.; Le, T.Y.; Gomez-Sanchez, C.E.; Morel-Kopp, M.C.; McWhinney, B.; Hudson, A.; Mihailidou, A.S. Role of Nongenomic Signaling Pathways Activated by Aldosterone During Cardiac Reperfusion Injury. Mol Endocrinol 2015, 29, 1144-1155. doi:10.1210/ME.2014-1410

24. Faresse, N. Post-translational modifications of the mineralocorticoid receptor: How to dress the receptor according to the circumstances? J Steroid Biochem Mol Biol 2014, 143, 334-342. doi:10.1016/j.jsbmb.2014.04.015

25. Fuller, P.J. Novel interactions of the mineralocorticoid receptor. Mol Cell Endocrinol 2015, 408, 33-37. doi: 10.1016/j.mce.2015.01.027

26. Oakley RH, Cruz-Topete D, He BO, Foley JF, Myers PH, Xu X , Gomez-Sanchez, C.E.; Chambon, P.; Willis, M.S.; Cidlowski, J.A. Cardiomyocyte glucocorticoid and mineralocorticoid receptors directly and antagonistically regulate heart disease in mice. Sci Signal 2019, 12, 577. doi:10.1126/scisignal.aau9685

27. Markan, U.; Pasupuleti, S.; Pollard, C.M.; Perez, A.; Lymperopoulos, A. The place of ARBs in heart failure therapy: is aldosterone suppression the key? Ther Adv Cardiovasc Dis 2019, 13, 1753944719868134. doi: $10.1177 / 1753944719868134$

28. Lymperopoulos, A.; Aukszi, B. Angiotensin receptor blocker drugs and inhibition of adrenal beta-arrestin-1-dependent aldosterone production: Implications for heart failure therapy. World J Cardiol 2017, 9, 200-206. doi: 10.4330/wjc.v9.i3.200

29. Juurlink, D.N.; Mamdani, M.M.; Lee, D.S.; Kopp, A.; Austin, P.C.; Laupacis, A.; Redelmeier, D.A. Rates of hyperkalemia after publication of the Randomized Aldactone Evaluation Study. N Engl J Med 2004, 351, 543-551. doi: 10.1056/NEJMoa040135

30. Shibata, S.; Rinehart, J.; Zhang, J.; Moeckel, G.; Castañeda-Bueno, M.; Stiegler, A.L.; Boggon, T.J.; Gamba, G.; Lifton, R.P. Mineralocorticoid receptor phosphorylation regulates ligand binding and renal response to volume depletion and hyperkalemia. Cell Metab 2013, 18, 660-671. doi: 10.1016/j.cmet.2013.10.005

31. Eijgelsheim, M.; Visser, L.E.; Uitterlinden, A.G.; Stricker, B.H. Protective effect of a GRK5 polymorphism on heart failure and its interaction with beta-adrenergic receptor antagonists. Pharmacogenomics 2008, 9, 1551-1555. doi: 10.2217/14622416.9.10.1551

32. Wu, J.H.; Zhang, L.; Fanaroff, A.C.; Cai, X.; Sharma, K.C.; Brian, L.; Exum, S.T.; Shenoy, S.K.; Peppel, K.; Freedman, N.J. G protein-coupled receptor kinase-5 attenuates atherosclerosis by regulating receptor tyrosine kinases and 7-transmembrane receptors. Arterioscler Thromb Vasc Biol 2012, 32, 308-316. doi:10.1161/ATVBAHA.111.239608

33. Montó, F.; Oliver, E.; Vicente, D.; Rueda, J.; Agüero, J.; Almenar, L.; Ivorra, M.D.; Barettino, D.; D'Ocon, P. Different expression of adrenoceptors and GRKs in the human myocardium depends on heart failure etiology and correlates to clinical variables. Am J Physiol Heart Circ Physiol 2012, 303, H368-H376. doi:10.1152/ajpheart.01061.2011 